\title{
Invasive micropapillary carcinoma of the extrahepatic bile duct and its malignant potential
}

\author{
TADASHI YOSHIZAWA ${ }^{1,2}$, YOSHIKAZU TOYOKI ${ }^{2}$, HIDEAKI HIRAI ${ }^{1}$, TOSHIHIRO HAGA ${ }^{1}$, TAKAHITO TOBA ${ }^{1}$, \\ SHINGO SAKURABA ${ }^{1,2}$, KENSUKE OKANO ${ }^{1,2}$, YUNYAN WU ${ }^{1}$, HIROKO SEINO ${ }^{1}$, \\ SATOKO MOROHASHI ${ }^{1}$, KENICHI HAKAMADA ${ }^{2}$ and HIROSHI KIJIMA ${ }^{1}$ \\ Departments of ${ }^{1}$ Pathology and Bioscience, and ${ }^{2}$ Surgery, Hirosaki University \\ Graduate School of Medicine, Hirosaki 036-8562, Japan
}

Received February 18, 2014; Accepted May 12, 2014

DOI: $10.3892 / o r .2014 .3394$

\begin{abstract}
Invasive micropapillary carcinoma (IMPC) was originally described as a distinctive type of invasive carcinoma in the breast, but it has not been recognized as a histological type of the extrahepatic bile duct cancer. The present study demonstrated clinicopathological features and patient prognosis of IMPC. We examined histological reviews of 93 consecutive cases of the extrahepatic bile duct cancer and identified 13 cases which included IMPC component. The component of IMPC ranged from 5 to $60 \%$ of the primary tumor tissue, which was mainly detected at the invasive front of the tumor. Of the 13 cases, $12(92.3 \%)$ carcinomas with IMPC showed lymph node metastasis more frequently compared to conventional adenocarcinoma $(39.2 \%, \mathrm{P}<0.001)$. Presence of IMPC component was significantly associated with poor overall survival $(\mathrm{P}=0.003)$. In conclusion, extrahepatic bile duct carcinoma with IMPC component showed significant lymphatic invasion, lymph node metastasis, and resulted in poor prognosis.
\end{abstract}

\section{Introduction}

Extrahepatic bile duct carcinoma is an epithelial cancer originating from the bile ducts with features of cholangiocytic differentiation. There is no significant geographical variation in the incidence of extrahepatic bile duct carcinoma. In the USA, extrahepatic bile duct carcinoma accounts for $0.16 \%$ of all invasive cancers in males and $0.15 \%$ in females in the general population (1). Surgical treatment is the only curative therapy for extrahepatic bile duct carcinoma and is therefore the treatment of choice if feasible. The spreading cancer cells via the lymphatic to regional lymph nodes are an important

Correspondence to: Dr Hiroshi Kijima, Department of Pathology and Bioscience, Hirosaki University Graduate School of Medicine, Zaifu-cho 5, Hirosaki 036-8562, Japan

E-mail: hkijima@cc.hirosaki-u.ac.jp

Key words: invasive micropapillary carcinoma, extrahepatic bile duct, lymphatic invasion, lymph node metastasis, prognosis factor for tumor progression. In a recent study, the median disease-specific survival rate after surgery in patients with lymph node metastasis was lower than that of patients without lymph node metastasis (19.3 vs. 53.5 months, $\mathrm{P}<0.0001)(2)$.

Recent studies have demonstrated that an invasive micropapillary carcinoma (IMPC) frequently shows aggressive tumor behaviors with marked lymph-vascular invasion, resulting in poor prognosis in several organs including the breast (3-5), urinary bladder (6-8), lung (9-13), parotid gland $(14,15)$, pancreas $(16)$, gallbladder $(17)$, colorectum (18) and stomach (19-22). To the best of our knowledge, however, there has been only one case report of extrahepatic bile duct carcinoma which contains IMPC (23). Therefore, the clinicopathological significance of IMPC has not yet been elucidated in extrahepatic bile duct. To clarify the significance of IMPC in extrahepatic bile duct, the present study investigated the clinicopathological features of 13 cases of extrahepatic bile duct carcinoma containing IMPC, compared with 80 cases of conventional extrahepatic bile duct carcinoma.

\section{Materials and methods}

Patients. We investigated consecutive bile duct carcinoma surgical cases treated between January 2007 and December 2012, after obtaining each patient's informed consent for use of their clinical records and pathological specimens at Hirosaki University Hospital. The series consisted of 69 men and 24 women with a median age of 70 years (range, 31-83 years). The carcinomas were located in the perihilar (34 cases) and distal bile duct (59 cases), according to the anatomic location (24). Curative resection and regional lymph node dissection were dependent on the location of primary tumors: pancreaticoduodenectomy or pylorus-preserving pancreaticoduodenectomy was performed in 56 patients, bile duct resection in 1 patient and combined hepatectomy with bile duct resection in 29 cases and combined hepatectomy and pancreaticoduodenectomy in 7 patients. Survival data were obtained from hospital medical charts and the median observation period was 25.6 months (79 cases).

Pathological analysis. All surgically resected specimens were routinely fixed with $10 \%$ formalin, then embedded in paraffin 
Table I. Extrahepatic bile duct cancer with IMPC component (13 cases).

\begin{tabular}{llcccl}
\hline $\begin{array}{l}\text { Case } \\
\text { No. }\end{array}$ & Age/gender & $\begin{array}{c}\text { Percentage of IMPC } \\
\text { component }\end{array}$ & $\begin{array}{c}\text { Lymph node } \\
\text { metastasis }\end{array}$ & $\begin{array}{c}\text { Survival } \\
\text { (months) }\end{array}$ & Status \\
\hline 1 & $74 / \mathrm{M}$ & $50-60$ & $9 / 17$ & 2 & Deceased \\
2 & $49 / \mathrm{F}$ & $40-50$ & $6 / 29$ & 54 & Deceased \\
3 & $71 / \mathrm{M}$ & $20-30$ & $2 / 6$ & N/A & N/A \\
4 & $70 / \mathrm{M}$ & $10-20$ & $2 / 6$ & 24 & Deceased \\
5 & $65 / \mathrm{M}$ & $10-20$ & $1 / 8$ & 7 & Deceased \\
6 & $70 / \mathrm{M}$ & $10-20$ & $1 / 10$ & 18 & Alive \\
7 & $72 / \mathrm{M}$ & $10-20$ & $3 / 23$ & N/A & N/A \\
8 & $72 / \mathrm{F}$ & $5-10$ & $2 / 21$ & 30 & Deceased \\
9 & $73 / \mathrm{M}$ & $5-10$ & $3 / 39$ & 8 & Deceased \\
10 & $73 / \mathrm{M}$ & $5-10$ & $0 / 19$ & 27 & Alive \\
11 & $43 / \mathrm{M}$ & $5-10$ & $1 / 10$ & 16 & Deceased \\
12 & $74 / \mathrm{M}$ & $5-10$ & $4 / 34$ & 9 & Deceased \\
13 & $80 / \mathrm{M}$ & $5-10$ & $1 / 14$ & N/A & N/A \\
\hline
\end{tabular}

IMPC, invasive micropapillary carcinoma; M, male; F, female; N/A, not applicable.

and stained with hematoxylin and eosin $(\mathrm{H} \& \mathrm{E})$ for pathological evaluation. The following histological features were assessed: depth of invasion (T-grade), histological type, lymphovascular invasion, perineural invasion, the mode of infiltration pattern, lymph nodal metastasis and IMPC component. We defined IMPC as tumor clusters of tumor cells lying within clear spaces. The clear spaces have no endothelial lining. Cases with tumor clusters only in the lymphovascular channels or mucinous lesions, which resemble the IMPC pattern, were excluded. We also evaluated the component ratio of IMPC in the entire tumor tissues. In cases where the distinction of lymphovascular invasion from the IMPC was difficult based on H\&E stained alone, we evaluated the cases with immunohistochemical podoplanin (D2-40) staining. Degrees of lymphatic, vessel and perineural invasion were classified as: 0 , no invasion; 1 , mild invasion; 2, moderate invasion; 3 , severe invasion. Modes of infiltration pattern were classified into three groups, i.e., INF- $\alpha$, cancer nests showing expansive growth and presenting the clear borderline between the tumor tissue and stroma; INF- $\beta$, intermediate patterns of growth and invasive structure of INF- $\alpha$ and INF- $\gamma$; and INF- $\gamma$, scirrhous growth with unclear borderline of the invasive front. These data were evaluated according to our previous study (25) and the General Rules for Surgical and Pathological Studies on Cancer of the Biliary Tract (26) with reference to the World Health Organization classification and staged according to the TMN classification of the International Union Against Cancer (UICC) (24). We also investigated phenotypes of IMPC components using the immunohistochemical procedure, as described below.

Immunohistochemistry. For histological examination, extrahepatic bile duct carcinoma specimens were routinely fixed with formalin, embedded in paraffin and thin-sectioned. Sections $4-\mu$ m-thick were mounted on saline-coated glass slides. Immunohistochemical examination was performed on deparaffinized sections using the standard avidin-biotin- peroxidase complex method with automated immunostainer (Benchmark XT; Ventana Medical System, Tucson, AZ, USA). The characteristic 'inside-out' pattern of IMPC was confirmed with the immunohistochemical MUC1 antibody. Furthermore, we investigated the phenotypes of IMPC using MUC1, MUC2, MUC5AC and MUC6 antibodies. Podoplanin (D2-40) was used for clarifying lymphatic invasion. The antibodies we used were: MUC1 (1:50, clone Ma696), MUC2 (1:50, clone Ccp), MUC5AC (1:100, clone CLH2), MUC6 (1:100, clone CLH5; all from Novocastra Laboratories), D2-40 (1:100, clone D2-40; Dako, Glostrup, Denmark).

Evaluation of immunohistochemistry. Luminal membranous immunoreactivities of the tumor were judged as positive for MUC1, and cytoplasmic immunoreactivities as positive for MUC2, MUC5AC and MUC6. The results were classified into two groups based on the percentage of positive cells with each staining as follows: negative group in which $<5 \%$ of cancer cells were stained, and positive group in which $\geq 5 \%$ was stained.

Statistical analysis. Statistical comparisons between two groups were analyzed using the Pearson's Chi-square test for categorical data and the Mann-Whitney test for continuous data. Survival curves were constructed using the Kaplan-Meier method and differences in survival were evaluated using the log-rank test. The relative prognostic factors were analyzed with the Cox proportional hazards regression model. Differences were considered to be statistically significant if the P-value was $<0.05$. All statistical evaluations were performed using R (http://www.r-project.org) and PASW statistics software (version 18.0; SPSS, Inc., Chicago, IL, USA).

\section{Results}

Histological and immunohistochemical findings of IMPC. We reviewed 93 cases of the extrahepatic bile duct cancer 

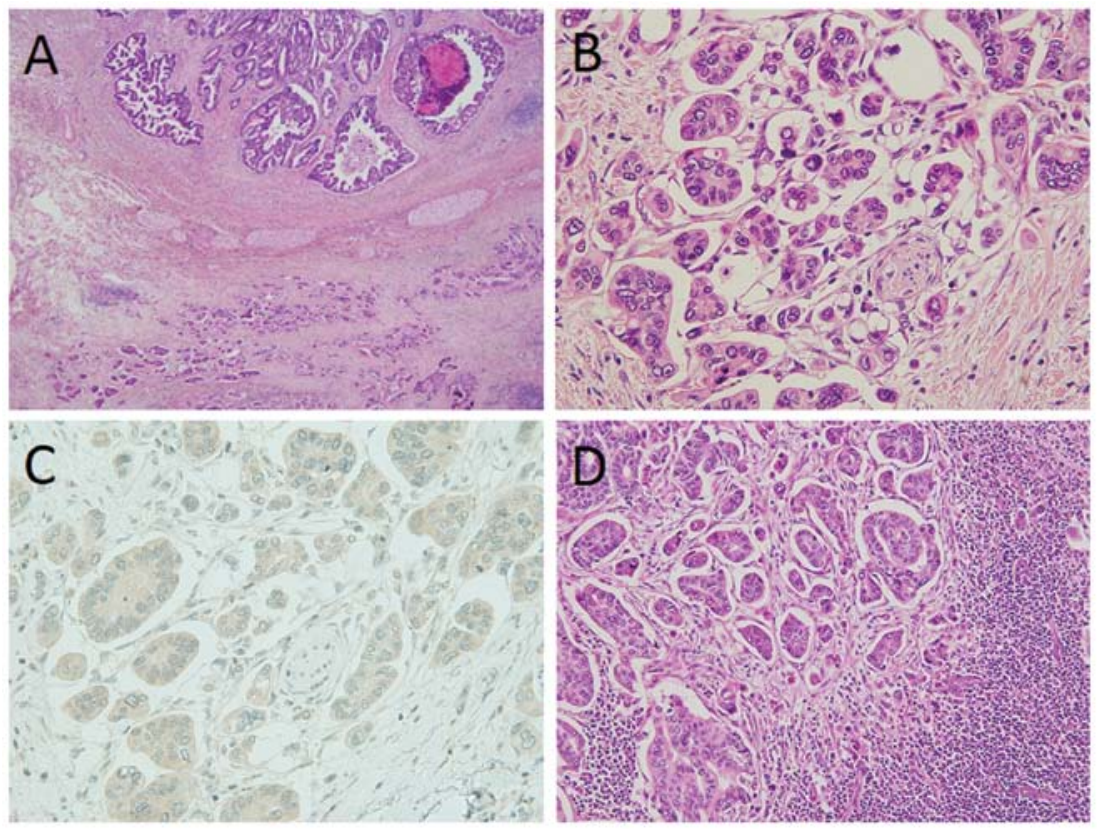

Figure 1. Histological findings of IMPC of the extrahepatic bile duct. IMPC component was found at the invasive front of the tumor (A, top left). Cancer cells were characterized by small round to ovoid micropapillary tumor cell clusters with no fibrovascular cores, lying within clear stromal spaces (B, top right). Immunohistochemical staining for D2-40 revealed the absence of endothelial lining cells in clear spaces surrounding the tumor clusters (C, bottom left). Lymph node metastasis also consisted of the IMPC (D, bottom right). The tumor clusters were similar to the primary lesion. IMPC, invasive micropapillary carcinoma.
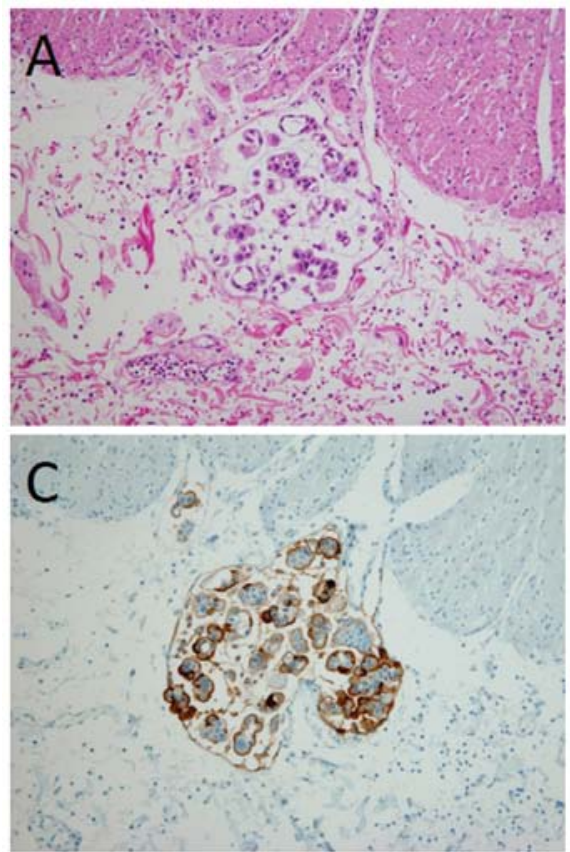

and found 13 cases (14.0\%) with IMPC component. The clinicopathological findings are summarized in Table I. The 13 patients (11 men and two women) with IMPC ranged in age from 49 to 80 years (mean, 68 years). Follow-up information was available for 10 patients, with a median follow-up time of 17 months (range, 2-54 months). Eight patients succumbed to the disease, one was alive with disease and one was alive without recurrent disease. Histologically, IMPC components ranging from 5 to $60 \%$ (mean $\pm \mathrm{SD}, 17.7 \pm 15.4$, data not shown) of the entire tumor were mainly found at the front of the

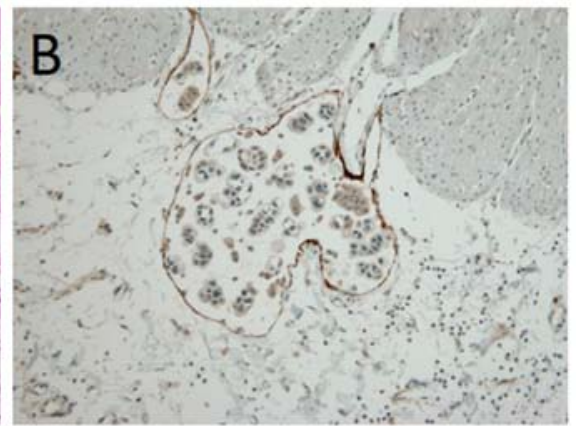

Figure 2. Marked lymphatic invasion of IMPC component was detected (A, top left), immunohistochemical staining for D2-40 revealed the endothelial lining cells (B, top right). Immunohistochemical staining for MUC1 revealed the 'inside-out' pattern (C, bottom left). IMPC, invasive micropapillary carcinoma.

tumor (Fig. 1A). The tumor was characterized by small round to ovoid micropapillary tumor cell clusters with no fibrovascular cores, lying within clear stromal spaces (Fig. 1B). The clear stromal spaces resembled lymphatic vessels, but were immunohistochemically negative for D2-40, a marker of lymphatic vessel (Fig. 1C). Metastatic carcinomas of lymph nodes also had IMPC component (Fig. 1D). The carcinoma cells characteristically displayed a reverse polarity, known as an 'inside-out' growth pattern, mimicking extensive lymphatic invasion (Fig. 2A and B). 

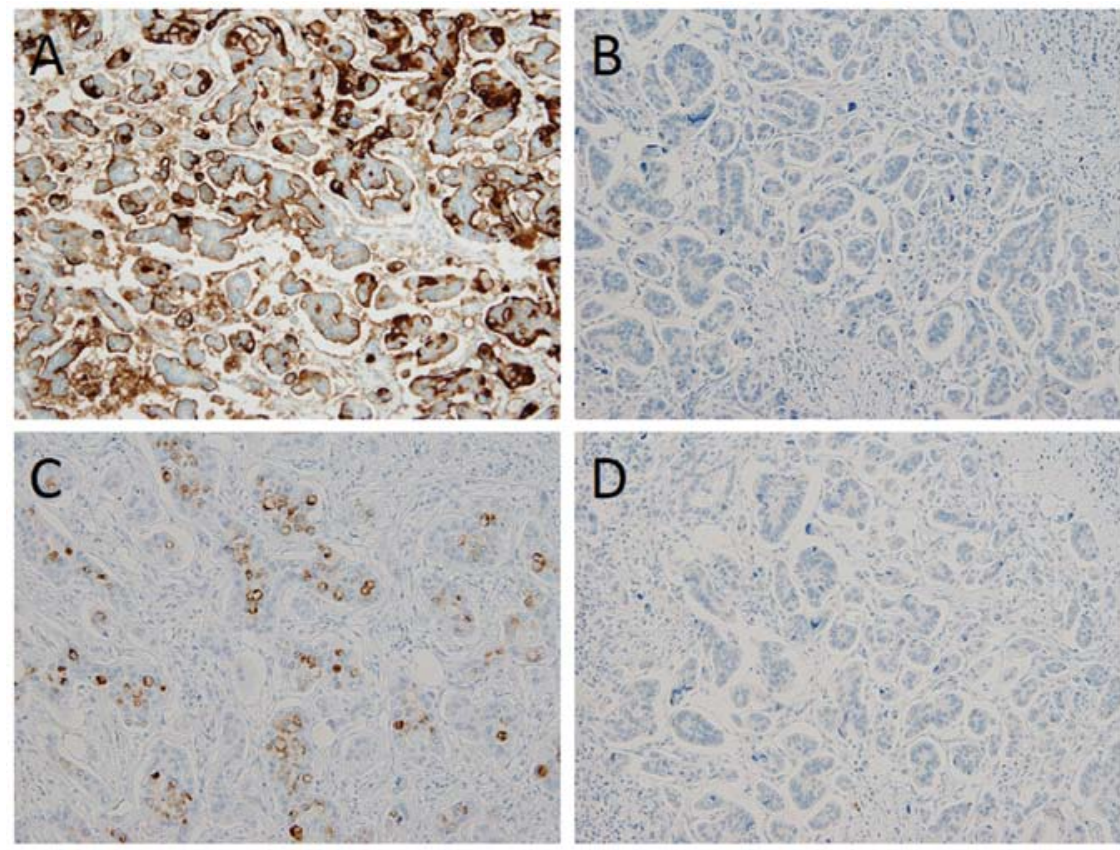

Figure 3. Immunohistochemical findings of a representative case. The 'inside-out' structures of IMPC were positive for MUC1 at peripheral cell membranes (A, top left), negative for MUC2 (B, top right), focally positive for MUC5AC (C, bottom left) and negative for MUC6 (D, bottom right). IMPC, invasive micropapillary carcinoma.

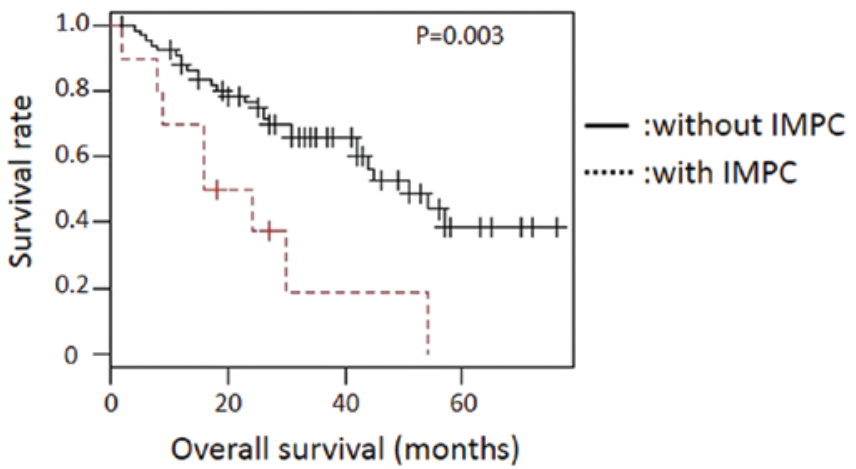

Figure 4. OS rate of patients with IMPC component. Patients with IMPC component showed reduced OS ( $\mathrm{P}=0.003)$. OS, overall survival; IMPC, invasive micropapillary carcinoma.

The results of immunohistochemistry are summarized in Table II. Nine (69.2\%) of the 13 cases of IMPC were positive for MUC1. MUC1 immunoreactivity was predominantly detected at the surface of the cell cluster and clearly exhibited the 'inside-out' growth pattern (Figs. 2C and 3A). MUC5AC was focally found in the cytoplasm, as well as at the cell surface, in 4 of the 13 cases (Fig. 3C). MUC2 and MUC6 staining was negative in all cases of IMPC (Fig. 3B and D).

Clinicopathological findings of IMPC. The clinicopathological findings of extrahepatic bile duct carcinoma with and without IMPC component are summarized in Table III. The presence of IMPC component was significantly correlated with lymph node metastasis, lymphatic invasion and the mode of infiltration pattern $(\mathrm{P}<0.001, \mathrm{P}=0.016$ and $\mathrm{P}=0.027$, respectively $)$. In addition, the extrahepatic bile duct cancer with IMPC compo-

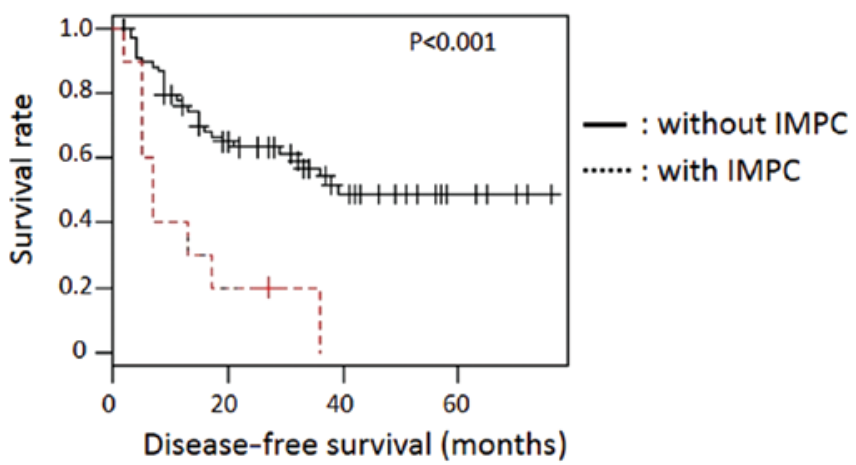

Figure 5. DFS rate of patients with IMPC component. Patients with IMPC component showed reduced DFS $(\mathrm{P}<0.001)$. DFS, disease-free survival; IMPC, invasive micropapillary carcinoma.

nent frequently showed lymph node metastasis with IMPC component (Table IV, $\mathrm{P}<0.001$ ).

Univariate and multivariate analysis. Survival curves based on univariate survival analysis demonstrated that the patients with IMPC were associated with poor prognosis (Fig. 4, $\mathrm{P}=0.003$ ) and poor disease-free survival (Fig. 5, $\mathrm{P}<0.001$ ). To clarify potential prognostic indicators, we analyzed various pathological factors investigated (Table V). Univariate analysis revealed that the following factors were correlated with poor prognosis: IMPC component [relative risk (RR) $3.195,95 \%$ confidence interval (CI) 1.437-7.107, $\mathrm{P}=0.004]$, depth of invasion (RR 3.261, 95\% CI 1.583-6.719, $\mathrm{P}=0.001$ ), histological type (RR 6.787, 95\% CI 2.072-22.23, $\mathrm{P}=0.002$ ), lymphatic invasion (RR 4.028, 95\% CI 1.973-8.224, $\mathrm{P}<0.001$ ), venous invasion (RR 2.714, 95\% CI 1.327-5.551, $\mathrm{P}=0.006$ ) 
Table II. Immunohistochemical characteristics of IMPC component of extrahepatic bile duct cancer (13 cases).

\begin{tabular}{lccccc}
\hline & & \multicolumn{5}{c}{ Mucin phenotype } \\
\cline { 3 - 6 } Case & Age/ & \multicolumn{5}{c}{ No. } & gender & MUC1 & MUC2 & MUC5AC & MUC6 \\
\hline 1 & $74 / \mathrm{M}$ & + & - & + & - \\
2 & $49 / \mathrm{F}$ & - & - & - & - \\
3 & $71 / \mathrm{M}$ & - & - & - & - \\
4 & $70 / \mathrm{M}$ & + & - & - & - \\
5 & $65 / \mathrm{M}$ & + & - & + & - \\
6 & $70 / \mathrm{M}$ & + & - & - & - \\
7 & $72 / \mathrm{M}$ & + & - & - & - \\
8 & $72 / \mathrm{F}$ & + & - & + & - \\
9 & $73 / \mathrm{M}$ & - & - & - & - \\
10 & $73 / \mathrm{M}$ & + & - & - & - \\
11 & $43 / \mathrm{M}$ & - & - & - & - \\
12 & $74 / \mathrm{M}$ & + & - & + & - \\
13 & $80 / \mathrm{M}$ & + & - & - & - \\
\hline
\end{tabular}

IMPC, invasive micropapillary carcinoma; M, male; F, female; Mucin phenotypes: MUC1, biliary; MUC2, intestinal; MUC5AC, gastric foveolar; MUC6, gastric pyloric.

and lymph node metastasis (RR 3.868, 95\% CI 1.946-7.684, $\mathrm{P}<0.001)$.

\section{Discussion}

In the present study, we clarified clinicopathological characteristics of IMPC of the extrahepatic bile duct. We clarified that IMPC frequently showed aggressive tumor growth with lymphatic invasion and lymph node metastasis, resulting in short overall/disease-free survival of the patients. This is the first report describing clinicopathological malignant potential of IMPC of the extrahepatic bile duct.

IMPC component has been reported in several organs, such as breast, urinary bladder, lung, parotid gland, pancreas, gallbladder, colorectum and stomach (3-22). The previous reports revealed that IMPC exhibited a tendency for lymphatic invasion and lymph node metastasis. In our study, IMPC component of extrahepatic bile duct ranged from 5 to $60 \%$ of the entire tumor, while with IMPC components were found in the other organs: $10-90 \%$ in salivary duct carcinoma (15), $10-90 \%$ in gastric carcinoma $(21), 5-10 \%$ in gallbladder carcinoma (17), 5-95\% in breast cancer (27). IMPC component, regardless of its tumor volume, has been shown to have malignant potential for lymphatic invasion and lymph node metastasis.

The molecular mechanisms of the malignant potential of IMPC have not yet been fully elucidated, while IMPC is histologically characterized by the 'inside-out' pattern. A previous study proposed that extracellular matrix (ECM) contributed to the IMPC structure (28). Madin-Darby canine kidney (MDCK) cells exhibited 'inside-out' structure without type I collagen, but were able to reorient their cell polarity under the
Table III. Histopathological characteristics of extrahepatic bile duct cancer with or without IMPC component.

\begin{tabular}{|c|c|c|c|}
\hline Variables & $\begin{array}{c}\text { With } \\
\text { IMPC } \\
\text { component } \\
(n=13)\end{array}$ & $\begin{array}{c}\text { Without } \\
\text { IMPC } \\
\text { component } \\
(\mathrm{n}=80)\end{array}$ & P-value \\
\hline Age & & & 0.956 \\
\hline$>65$ & 3 & 58 & \\
\hline$\leq 65$ & 10 & 22 & \\
\hline Gender & & & 0.503 \\
\hline Male & 11 & 58 & \\
\hline Female & 2 & 22 & \\
\hline Location & & & 0.361 \\
\hline Perihilar $^{\mathrm{a}}$ & 3 & 31 & \\
\hline Distal $^{\mathrm{a}}$ & 10 & 49 & \\
\hline Depth of invasion & & & 0.071 \\
\hline $\mathrm{T} 1$ or $\mathrm{T} 2^{\mathrm{b}}$ & 3 & 40 & \\
\hline $\mathrm{T} 3$ or $^{\mathrm{T}} 4^{\mathrm{b}}$ & 10 & 40 & \\
\hline Histological type & & & 0.332 \\
\hline $\begin{array}{l}\text { Well-differentiated } \\
\text { adenocarcinoma }\end{array}$ & 2 & 27 & \\
\hline Other histological type & 11 & 53 & \\
\hline Lymph node metastasis & & & $<0.001$ \\
\hline $\mathrm{pN}(+)$ & 12 & 31 & \\
\hline Lymphatic invasion & & & 0.016 \\
\hline ly0 or ly 1 & 2 & 41 & \\
\hline ly 2 or $1 y 3$ & 11 & 39 & \\
\hline Venous invasion & & & 0.063 \\
\hline v0 or v1 & 2 & 34 & \\
\hline v2 or v3 & 11 & 46 & \\
\hline Perineural invasion & & & 0.332 \\
\hline $\mathrm{n} 0$ or $\mathrm{n} 1$ & 2 & 27 & \\
\hline $\mathrm{n} 2$ or $\mathrm{n} 3$ & 11 & 53 & \\
\hline $\mathrm{INF}^{\mathrm{c}}$ & & & 0.027 \\
\hline$\alpha$ or $\beta$ & 1 & 34 & \\
\hline$\gamma$ & 12 & 46 & \\
\hline
\end{tabular}

IMPC, invasive micropapillary carcinoma; ${ }^{a}$ according to WHO classification; ${ }^{b}$ according to TNM classification; ' mode of infiltration pattern, as described in Materials and methods.

presence of type I collagen in ECM. Here, the reorientation of cell polarity was shown to be related to RAC1, PI3-kinase and a PKC (28). Cancer cells of IMPC may have abnormalities of RAC1 suppression cascade and show the characteristic 'inside-out' structures (29). We also revealed that MUC1 expression was predominantly at the surface of tumor clusters. The MUC1 expression was similar to the other organs (30). Human MUC1 is a high molecular weight transmembrane glycoprotein, which is apically expressed in the majority of glandular epithelia (31). Increased MUC1 expression has been shown to inhibit integrin-mediated cell adhesion between 
Table IV. Histological relationship between primary tumor and lymph node metastasis with or without IMPC component.

$\begin{array}{cc}\text { With IMPC } & \text { Without IMPC } \\ \text { component }(n=13) & \text { component }(n=80)\end{array} \quad$ P-value

Lymph node status

$\mathrm{pN}(+)$

$12(92.3 \%)$

$31(38.8 \%)$

$<0.001$

\begin{tabular}{lcc}
\hline & $\begin{array}{c}\text { Primary with IMPC } \\
\text { component }(\%)\end{array}$ & $\begin{array}{c}\text { Primary without IMPC } \\
\text { component }(\%)\end{array}$ \\
\hline Lymph node with IMPC component & $11(25.6)$ & $1(2.3)$ \\
Lymph node without IMPC component & $1(2.3)$ & $30(69.8)$ \\
\hline
\end{tabular}

IMPC, invasive micropapillary carcinoma.

Table V. Univariate and multivariate analysis of prognostic factors of survival.

\begin{tabular}{|c|c|c|c|}
\hline Variables & Values (\%) & $\begin{array}{c}\text { Univariate analysis } \\
\text { P-value }\end{array}$ & $\begin{array}{c}\text { Multivariate analysis } \\
\text { P-value }\end{array}$ \\
\hline IMPC component & & 0.004 & 0.643 \\
\hline With IMPC component & $10(12.7)$ & & \\
\hline Without IMPC component & $69(87.3)$ & & \\
\hline Depth of invasion & & 0.001 & 0.234 \\
\hline $\mathrm{T} 1$ or $\mathrm{T} 2^{\mathrm{a}}$ & $40(50.6)$ & & \\
\hline $\mathrm{T} 3$ or $\mathrm{T}^{\mathrm{a}}$ & $39(49.6)$ & & \\
\hline Histological type & & 0.002 & 0.062 \\
\hline Well-differentiated adenocarcinoma & $24(30.1)$ & & \\
\hline Other histological type & $55(69.6)$ & & \\
\hline Lymphatic invasion & & $<0.001$ & 0.223 \\
\hline ly0 or ly1 & $43(54.4)$ & & \\
\hline ly 2 or $1 y 3$ & $36(45.6)$ & & \\
\hline Venous invasion & & 0.006 & 0.76 \\
\hline v0 or v1 & $36(45.6)$ & & \\
\hline v2 or v3 & $43(54.4)$ & & \\
\hline Perineural invasion & & 0.071 & - \\
\hline $\mathrm{n} 0$ or $\mathrm{n} 1$ & $29(36.7)$ & & \\
\hline $\mathrm{n} 2$ or $\mathrm{n} 3$ & $50(63.3)$ & & \\
\hline $\mathrm{INF}^{\mathrm{b}}$ & & 0.064 & - \\
\hline$\alpha$ or $\beta$ & $30(38.0)$ & & \\
\hline$\gamma$ & $49(62.0)$ & & \\
\hline Lymph node metastasis & & $<0.001$ & 0.116 \\
\hline pN (-) & $50(63.3)$ & & \\
\hline $\mathrm{pN}(+)$ & $29(36.7)$ & & \\
\hline
\end{tabular}

IMPC, invasive micropapillary carcinoma; according to TNM classification; ${ }^{b}$ mode of infiltration pattern, as described in Materials and methods.

cancer cells and ECM (32) and to decrease adhesion to type I collagen (33). Based on this evidence, IMPC expressing MUC1 may reduce the cell adhesion from ECM and result in forming the characteristic 'inside-out' structures.
Our study revealed that IMPC structures were found not only in the primary carcinoma lesions, but also in the foci of lymphatic vessels and metastatic lymph nodes. The 'inside-out' IMPC structures were thought to play an important role in 
the lymph node metastasis. Of note, IMPC exhibited stromal desmoplastic reactions around the 'inside-out' cancer cell clusters. The desmoplastic changes consisted of proliferation of fibroblasts and collagen fibers and were found not only in the primary lesion, but also in the parts of lymph node metastasis. The desmoplastic changes are thought to be associated with epithelial-mesenchymal transition, which may contribute to an aggressive growth of the invasive cancer.

The results of univariate analysis revealed that IMPC was significantly correlated with poor patient prognosis, but the multivariate analysis using Cox proportional hazards model showed that IMPC was not an independent prognostic factor for overall survival. We suspected the reason why it was not an independent factor was that IMPC may be strongly associated with lymphatic invasion and lymph node metastasis. An important clinical issue is that the presence of IMPC indicates malignant potential, even if the component is small. Therefore, pathologists should describe presence of IMPC component in the diagnostic report, even if the component is a small part of the extrahepatic bile duct carcinoma.

\section{Acknowledgements}

This study was supported by Grants-in Aid for Science from the Ministry of Education, Culture, Sports, Science and Technology in Japan, and a Grant for Hirosaki University Institutional Research.

\section{References}

1. Fred T. Bosman and Fatima Carnerio (eds): World health Organization Classification of Tumours of the Digestive System. IARC Press, Lyon, pp272-273, 2012.

2. Ito K, Ito H, Allen PJ, et al: Adequate lymph node assessment for extrahepatic bile duct adenocarcinoma. Ann Surg 251: 675-681, 2010.

3. Tavassoli FA and Devilee P (eds): World Health Organization Classification of Tumours of the Breast and Female Genital Organs. IARC Press, Lyon, pp35-36, 2003.

4. Siriaunkgul S and Tavassoli FA: Invasive micropapillary carcinoma of the breast. Mod Pathol 6: 660-662, 1993.

5. Nassar H, Wallis T, Andea A, Dey J, Adsay V and Visscher D: Clinicopathologic analysis of invasive micropapillary differentiation in breast carcinoma. Mod Pathol 14: 836-841, 2001.

6. Lopez-Beltran A, Montironi R, Blanca A and Cheng L: Invasive micropapillary urothelial carcinoma of the bladder. Hum Pathol 41: 1159-1164, 2010.

7. Perepletchikov AM and Parwani AV: Micropapillary urothelial carcinoma: clinico-pathologic review. Pathol Res Pract 205: 807-810, 2009.

8. Samaratunga $\mathrm{H}$ and Khoo K: Micropapillary variant of urothelial carcinoma of the urinary bladder; a clinicopathological and immunohistochemical study. Histopathology 45: 55-64, 2004.

9. Kuroda N, Hamaguchi N, Takeuchi E, Ohara M, Hirouchi T and Mizuno K: Lung adenocarcinoma with a micropapillary pattern: a clinicopathological study of 25 cases. APMIS 11: 381-385, 2006.

10. Kuroda N, Hamaguchi N, Ohara M, Hirouchi T, Miyzaki E and Mizuno K: Intracytoplasmic lumina in invasive micropapillary carcinoma of the lung. Diagn Cytopathol 34: 224-226, 2006.

11. Kuroda N, Hamauzu T, Toi M, et al: Pulmonary adenocarcinoma with micropapillary component: an immunohistochemical study. Case report. APMIS 113: 550-554, 2005.
12. Makimoto Y, Nabeshima K, Iwasaki H, et al: Micropapillary pattern: a distinct pathological marker to subclassify tumours with a significantly poor prognosis within small peripheral lung adenocarcinoma $(</=20 \mathrm{~mm})$ with mixed bronchioloalveolar and invasive subtypes (Noguchi's type C tumours). Histopathology 46: 677-684, 2005.

13. Amin MB, Tamboli P, Merchant SH, et al: Micropapillary component in lung adenocarcinoma: a distinctive histologic feature with possible prognostic significance. Am J Surg Pathol 26: 358-364, 2002.

14. Yamamoto H, Uryu H, Segawa Y and Tsuneyoshi M: Aggressive invasive micropapillary salivary duct carcinoma of the parotid gland. Pathol Int 58: 322-326, 2008.

15. Nagao T, Gaffey TA, Visscher DW, et al: Invasive micropapillary salivary duct carcinoma: a distinct histologic variant with biologic significance. Am J Surg Pathol 28: 319-326, 2004.

16. Khayyata S, Basturk O and Adsay NV: Invasive micropapillary carcinomas of the ampullo-pancreatobiliary region and their association with tumor-infiltrating neutrophils. Mod pathol 18: 1504-1511, 2005.

17. Hara S, Kijima H, Okada K and Igarashi Y: Invasive micropapillary variant of the gallbladder adenocarcinoma and its aggressive potential for lymph node metastasis. Biomed Res 31: 89-95, 2010.

18. Kim MJ, Hong SM, Jang SJ, et al: Invasive colorectal micropapillary carcinoma: an aggressive variant of adenocarcinoma. Hum Pathol 37: 809-815, 2006.

19. Fujita T, Gotohda N, Kato Y, et al: Clinicopathological features of stomach cancer with invasive micropapillary component. Gastric cancer 15: 179-187, 2012.

20. Ohtsuki Y, Kuroda N, Yunoki S, et al: Immunohistochemical analysis of invasive micropapillary carcinoma pattern in four cases of gastric cancer. Med Mol Morphol 46: 114-121, 2013.

21. Ushiku T, Matsusaka K, Iwasaki Y, et al: Gastric carcinoma with invasive micropapillary pattern and its association with lymph node metastasis. Histopathology 59: 1081-1089, 2011.

22. Shimoda M, Okada $Y$, Hayashi $Y$, et al: Primary invasive micropapillary carcinoma of the stomach. Pathol Int 58: 513-517, 2008.

23. Kondo T: Bile duct adenocarcinoma with minor micropapillary component: a case report. Cases J 2: 51, 2009.

24. Sobin LH, Gospodarowicz MK and Wittekind CH (eds): TMN Classification of Malignant Tumors. 7th edition. Wiley-Liss, New York, 2009.

25. Okada K, Kijima H, Imaizumi T, et al: Clinical significance of wall invasion pattern of subserosa-invasive gallbladder carcinoma. Oncol Rep 28: 1531-1536, 2012.

26. Japanese Society of Biliary Surgery: General Rules for the Surgical and Pathological Studies on Cancer of the Biliary Tract. 5th edition. Kanehara Shuppan, Tokyo, 2003.

27. Walsh MM and Bleiweiss IJ: Invasive micropapillary carcinoma of the breast: eighty cases of an underrecognized entity. Hum Pathol 32: 583-589, 2001.

28. Liu KD, Datta A, Yu W, et al: Rac1 is required for reorientation of polarity and lumen formation through a PI 3-kinase-dependent pathway. Am J Physiol Renal Physiol 293: F1633-F1640, 2007.

29. Sahai E and Marshall CJ: RHO-GTPases and cancer. Nat Rev Cancer 2: 133-142, 2002.

30. Nassar H, Pansare V, Zhang H, et al: Pathogenesis of invasive micropapillary carcinoma: role of MUC1 glycoprotein. Mod Pathol 17: 1045-1050, 2004

31. Winterford CM, Walsh MD, Leggett BA and Jass JR: Ultrastructural localization of epithelial mucin core proteins in colorectal tissues. J Histochem Cytochem 47: 1063-1074, 1999.

32. Wesseling J, van der Valk SW, Vos HL, Sonnenberg A and Hilkens J: Episialin (MUC1) overexpression inhibits integrinmediated cell adhesion to extracellular matrix components. J Cell Biol 129: 255-265, 1995.

33. Hudson MJ, Stamp GW, Chaudhary KS, et al: Human MUC1 mucin: a potent glandular morphogen. J Pathol 194: 373-383, 2001. 